\title{
Meine sehr geehrten Damen und Herren!
}

Mikroelektronik ist aus unserer heutigen hoch technologisierten Welt nicht mehr wegzudenken und stellt die Basis für eine Vielzahl neuer und verbesserter Produkte dar. Die Mikroelektronik und deren Weiterentwicklung als Basis neuer innovativer Produkte ist damit auch ein wesentlicher Wirtschaftsfaktor.

Diese hohe wirtschaftliche Bedeutung spiegelt sich auch in Österreich wider. So beträgt alleine der Anteil der elektronischen Bauelemente knapp $17 \%$ an der Gesamtproduktion der österreichischen Elektro- und Elektronikindustrie und ist damit die größte produzierende Branche. Wenngleich der Produktionswert von elektronischen Bauelementen, bedingt durch schwache Nachfrage und teilweise dramatischen Preisverfall, gegenüber dem Vorjahr um fast $12 \%$ sank, konnte sich die heimische Industrie durch ihre Ausrichtung auf Spezialprodukte und verstärkten Export im internationalen Umfeld gut behaupten.

Betrachtet man die globale Entwicklung auf diesem Sektor, so sind nennenswerte Zuwächse derzeit nur in der Region Südostasien und China zu beobachten, während die Situation in Europa und den USA durch Stagnation und negatives Marktwachstum gekennzeichnet ist.

Mittelfristig wird es daher darum gehen, wieviel Wertschöpfung im globalen Umfeld in Europa und damit in Österreich gehalten werden kann. Erfolgreich werden hier in Zukunft nur Unternehmen sein, die über Innovationen im Produkt- und Produktionsbereich neue und bessere Produkte und Dienstleistungen auf den Markt bringen als ihr Mitbewerb.

Voraussetzung dafür ist ein leistungsfähiges effizientes Innovationssystem, als Basis einer nachhaltigen Wirtschaftsentwicklung. Auf europäischer Ebene wurde die Wichtigkeit von Forschung, Entwicklung und Innovation erkannt und im Rahmen des Rates von Lissabon eine Forschungsquote von $3 \%$ des BIP im Jahre 2010 als visionäres Ziel formuliert.

In Österreich hat der Rat für Forschung und Technologieentwicklung seine Emplehlungen für ein konsistentes, nachhaltiges Innovationssystem im nationalen Forschungs- und Innovationsplan Ende des vergangenen Jahres vorgestellt.

Als eines der wesentlichen Kriterien wird hier die Kooperation von Grundlagenforschung, angewandter Forschung sowie industrieller Forschung und Entwicklung im Rahmen von Netzwerken hervorgehoben. Ein Anspruch, dem die Mikroelektronik-

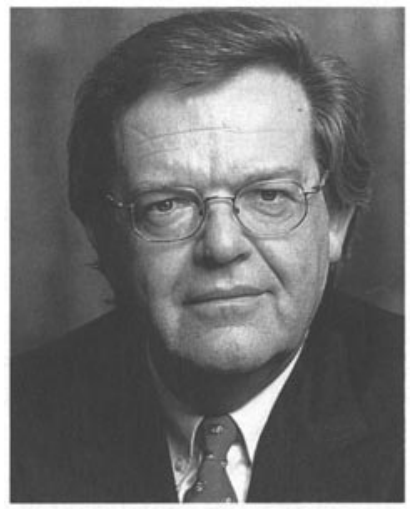

Dipl.-Ing. Albert Hochleitner

tagung traditionell gerecht wird, bietet sie doch allen Interessierten aus Wissenschaft und Wirtschaft die Möglichkeit, sich über die neuesten Entwicklungen und Trends zu informieren und mit den Tagungsteilnehmern zu diskutieren.

Mit der Konzentration auf die drei Themenbereiche Nanotechnologie, Embedded Systems und Wireless Access wurden sehr attraktive Forschungsgebiete mit hohem Zukunftspotenzial für innovative Produkte und Dienstleistungen ausgewählt. Zur Forcierung der nationalen Forschungs- und Entwicklungsaktivitäten bedarf es allerdings auch einer zielgerichteten öffentlichen Förderung mit einer zumindest mittelfristigen Planungssicherheit für Projekte und Programme. Die Mikroelektroniktagung 2003 ist sicher geeignet, hier entsprechende Impulse aus wissenschaftlicher und technischer Sicht zu geben.

In diesem Sinne möchte ich im Namen des Fachverbandes der Elektro- und Elektronikindustrie und seiner Mitgliedsunternehmen den Organisatoren danken, denen es auch heuer wieder gelungen ist, ein attraktives Programm zu gestalten, und wünsche den Teilnehmern eine interessante und anregende Veranstaltung.

Generaldirektor Dipl.-Ing. Albert Hochleitner

Vorsteher des Fachverbandes der Elektro- und Elektronikindustrie 